\title{
The Role of Qatar Community Pharmacists in Depression care: A Survey of Attitudes, Practices and Perceived Barriers
}

\author{
Rula Shami $^{1}$, Mohammed Fasihul Alam ${ }^{1}$, Maguy El Hajj ${ }^{2}$ \\ ${ }^{1}$ College of Health Sciences, Public health Department, Qatar University, Doha, Qatar ${ }^{2}$ College of Pharmacy, Qatar University, Doha, Qatar
}

\section{Background and Objective}

- Depression is a main contributor to mortality and exacerbation of other health problems [1], and one of the leading causes of disability in the world [2]. Negative attitudes and stigma remain to be major barriers to healthcare provision and access to treatment for patients with depression.

- Up to $33 \%$ of patients who have depression prematurely discontinue treatment within the first 30 days, and $40 \%$ of them within 90 days of antidepressant initiation [3].

- Community pharmacists play an important role in the care of major depression, being among the most accessible healthcare providers who can enhance patients' adherence to antidepressant regimens through antidepressant medication counselling and management [4]

- Currently Qatar has around 1200 community pharmacists, but little is known whether they are sufficiently contributing to depression care.

- Our study aims to describe the current practices, attitudes and perceived barriers of Qatar community pharmacists towards the provision of depression care, and to investigate how pharmacists' attitudes, along with several sociodemographic and professional characteristics, are associated with these practices.

\section{Methodology}

- A cross-sectional online survey targeting all practicing community pharmacists based on an updated contact list of practicing from Qatar Ministry of Public Health (MoPH) and using an adapted survey instrument.

Study outcomes were:

- scores of attitudes towards depression

scores of self-reported depression care practices

perceived barriers for depression care

- Responses were measured on a 5-point-Likert scale.

- Descriptive univariate and bivariate analyses of study outcomes were conducted, along with a multivariate regression, to investigate how pharmacists' characteristics and attitudes affect their practice.

- Data was analyzed using STATA 15.1 software

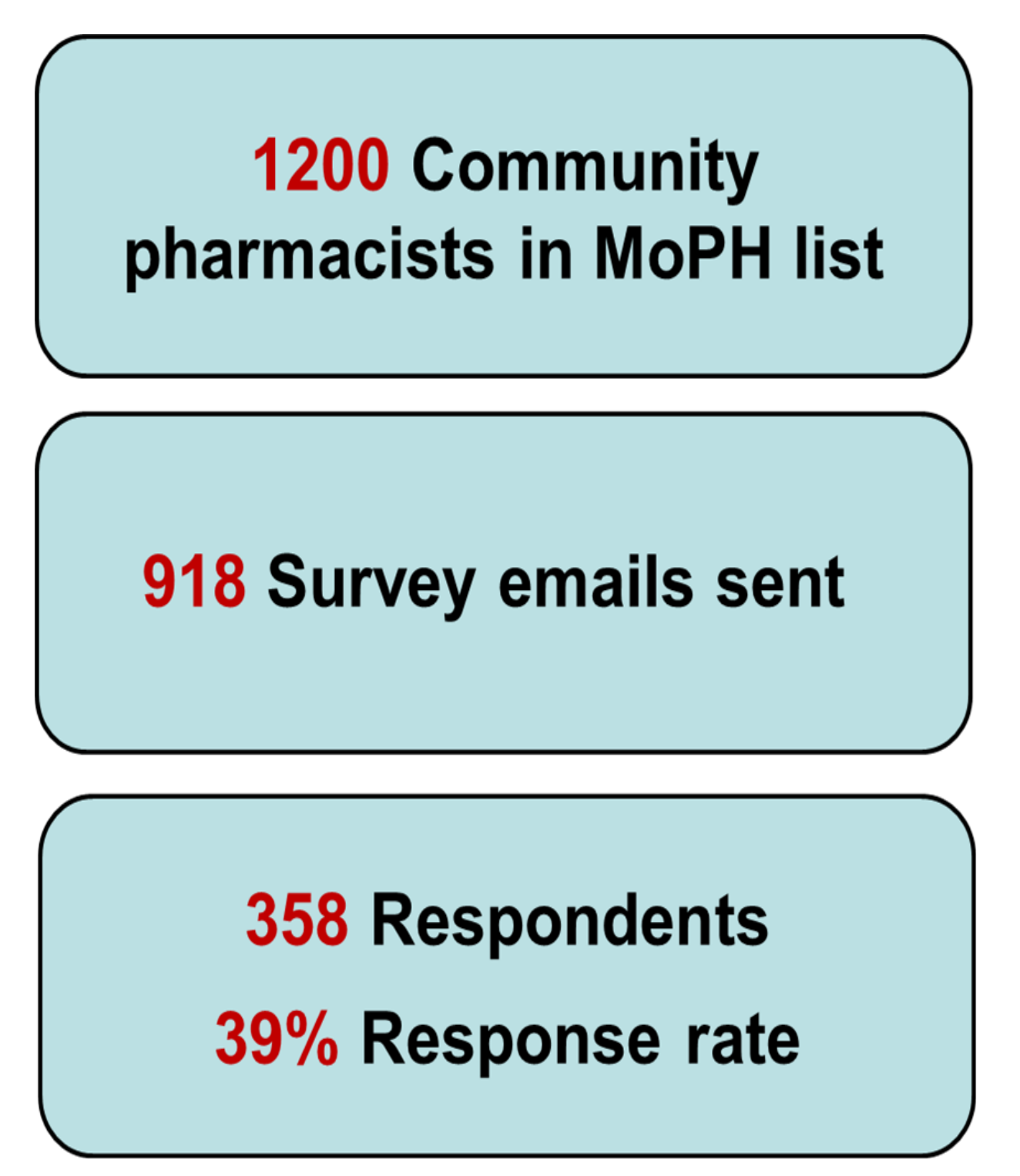

\section{Results: Pharmacists' Practices}

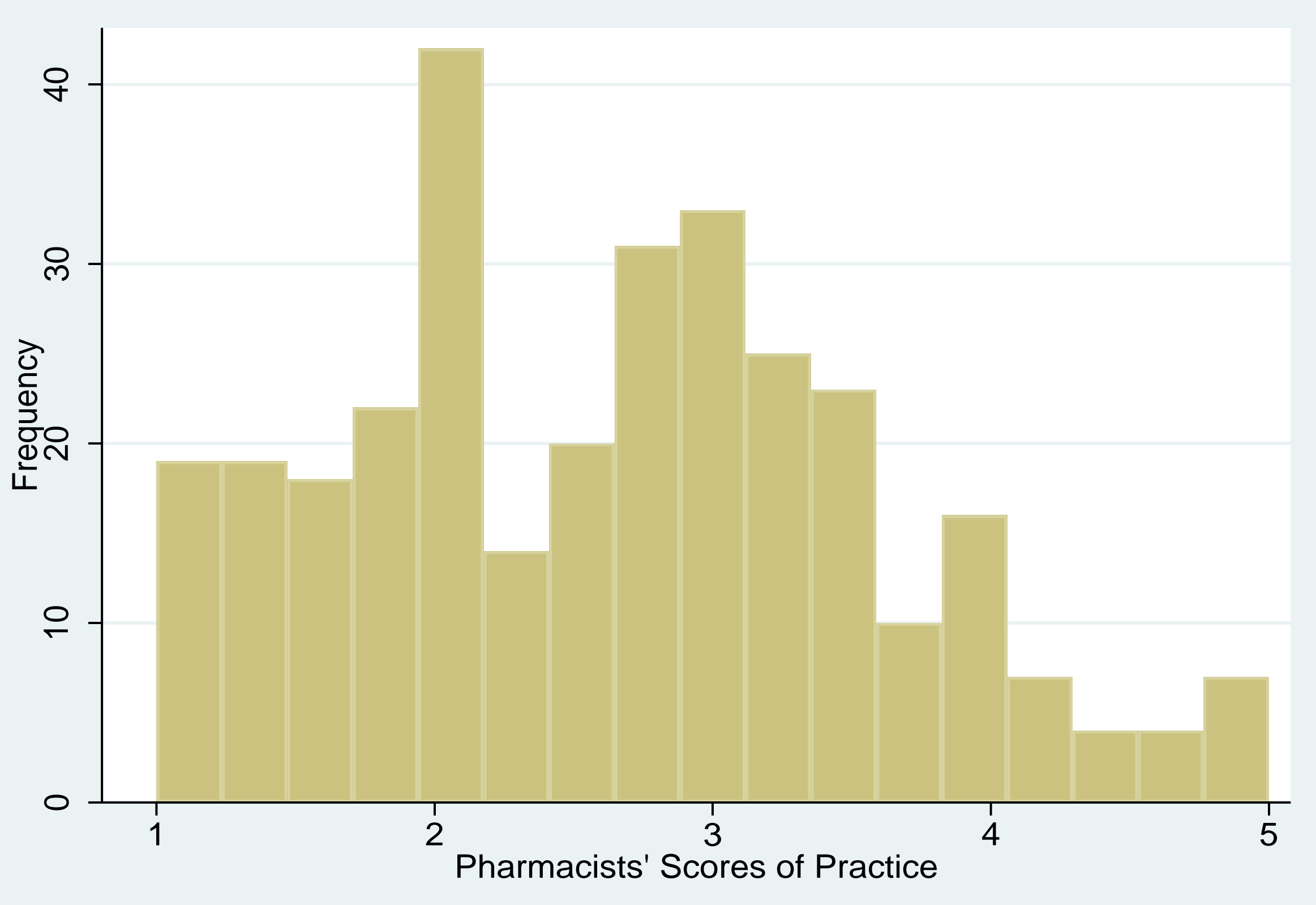

Figure 1:

Distribution of scores of practice

$\mathrm{N}=314$

Minimum $=1$

Maximum $=5$

Mean $=2.64$

$S D=0.94$

Cronbach Alpha $=0.94$

1= never 2= rarely

$3=$ sometimes $4=$ usually

$5=$ always

\section{Results: Pharmacists' Attitudes}

Figure 2:

Distribution of scores of attitude

$\mathrm{N}=263$

Minimum $=2.46$

Maximum $=4.18$

Mean $=3.41$

$\mathrm{SD}=0.26$

Cronbach Alpha $=0.90$

1= strongly disagree

2= disagree $3=$ neutral

4= agree 5= strongly agree

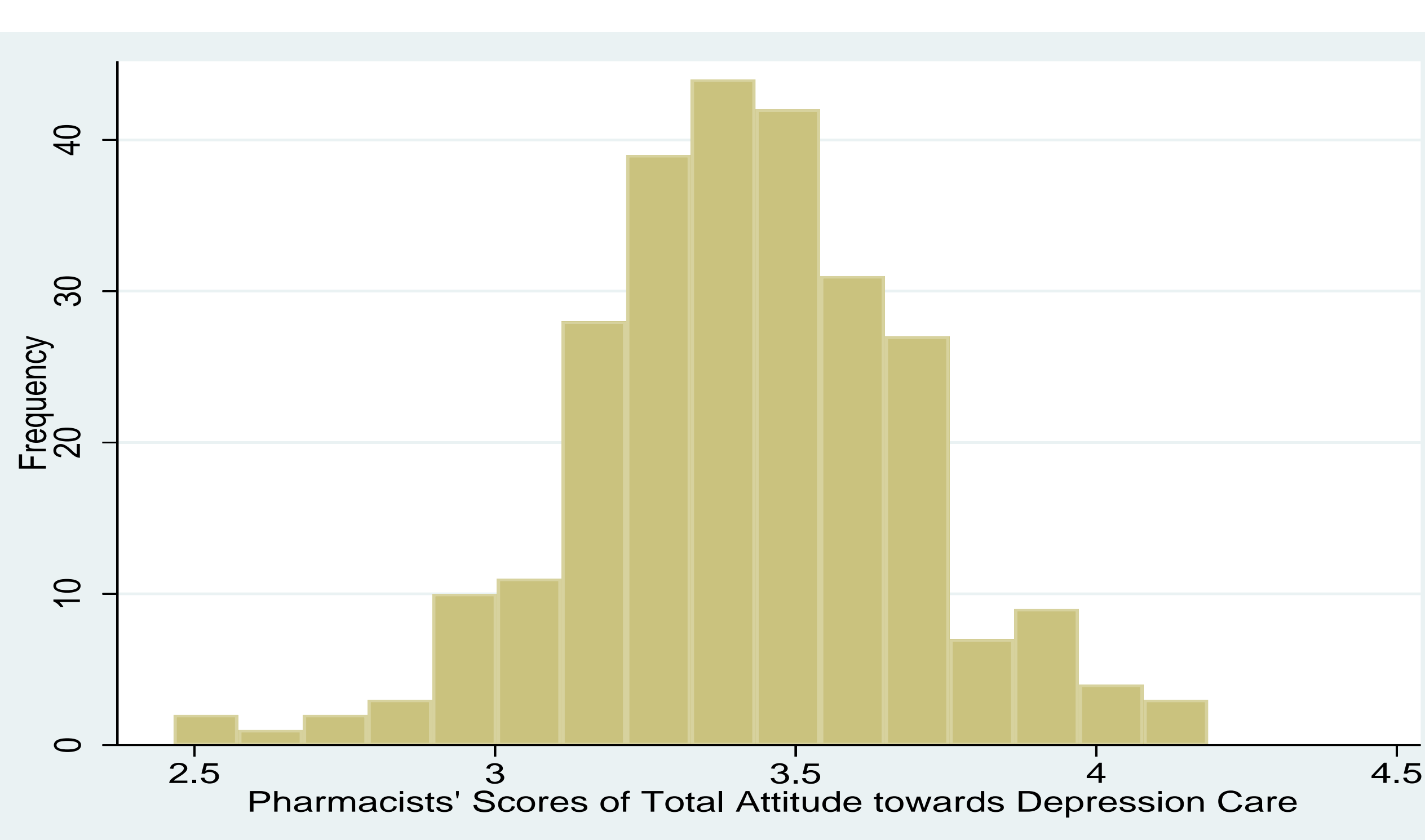

Pharmacists who "agreed "or "strongly agreed" to below statements:

I have adequate knowledge on medication therapy for major depression.

am confident about my ability to counsel patients with major depression on the importance $\quad 65.7 \%$ of adhering to their prescribed antidepressant medications

I am comfortable to counsel patients with major depression on the importance of adhering to $\mathbf{7 3 . 2 \%}$ their prescribed antidepressant medications

am interested to counsel patients with major depression on the importance of adhering to their prescribed antidepressant medications

It is ethical for me to tell the patient what the purpose of antidepressant drug is.

$75.9 \%$

I should advise patients on all potential side effects of antidepressant medications.

$77.5 \%$

There is a lot that patients can do to control symptoms

Anyone can suffer from major depression

I should actively support the recovery efforts made by individuals who suffer from major depression

Major depression has major consequences on life

$77.8 \%$

$76.3 \%$

$81.9 \%$

$84.3 \%$

$87.6 \%$

\section{Results :Pharmacists' Perceived Barriers}

Perceived barriers reported by pharmacists: $(\mathrm{N}=\mathbf{2 7 5})$

I am sensitive to ethnic/cultural differences

Shortage of personnel

Lack of clear clinical guidelines

Lack of needed time

Lack of a private setting in the pharmacy

Lack of needed knowledge and training on mental health

Lack of patient's insight on major depression and the importance of treatment

Lack of access to patients' medical profiles

\section{Results: Factors associated with pharmacists' practices}

- Female pharmacists were significantly less involved in depression care compared to male pharmacists (Beta-coefficient $=-4.36,95 \% \mathrm{Cl}-7.46$ to $-1.26, p<0.01$ )

- The presence of a private area for counselling patients was associated with higher scores of practice (Beta-coefficient=3.39, 95\% Cl 0.20 to $6.59 \mathrm{p}=0.03$ )

- Depression practice score increased with an increasing score of attitudes (Beta-coefficient $=0.22$ $95 \% \mathrm{Cl} 0.09$ to $0.35, p=0.001)$, and decreased with the number of years since the last pharmacy degree graduation $(p=0.02)$

\section{Conclusion}

- Pharmacists' moderately positive attitudes towards depression and its care were not reflected in their current practices. Such results could be explained by the presence of a set of perceived barriers that were identified in this study, such as the lack of needed knowledge and the lack of a private consultation area in the pharmacy.

- Study results imply a need for the development of depression care training programs that are directed to improve pharmacists' knowledge on depression and to reduce pharmacists' stigma towards patients who suffer from depression.

- Results from this study may also serve as a baseline for future local longitudinal studies addressed towards improving the provision of care for depression as a major public health issue in Qatar.

\section{References}

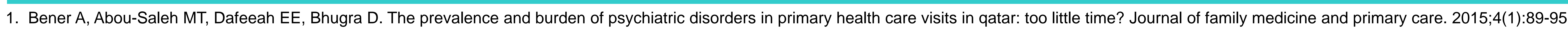

